\title{
A CANONICAL DISTRIBUTION ON ISOPARAMETRIC SUBMANIFOLDS I
}

\author{
CRISTIÁN U. SÁNCHEZ
}

\begin{abstract}
We show that on every compact, connected homogeneous isoparametric submanifold $M$ of codimension $h \geq 2$ in a Euclidean space, there exists a canonical distribution which is bracket generating of step 2. An interesting consequence of this fact is also indicated. In this first part we consider only the case in which the system of restricted roots is reduced, reserving for a second part the case of non-reduced restricted roots.
\end{abstract}

\section{INTRODUCTION}

This paper, of which we present here its first part, is devoted to indicating some properties (that we think have not been previously studied) of compact, connected homogeneous isoparametric submanifolds of Euclidean spaces of codimension $h \geq 2$.

By a celebrated theorem due to G. Thorbergsson [7], all compact, connected, isoparametric submanifolds of Euclidean spaces of codimension $h \geq 3$ are homogeneous. On the other hand, in codimension $h=2$ there are infinitely many non-homogeneous examples and only a finite number of homogeneous ones. Homogeneous isoparametric submanifolds $M^{n}$ of $\mathbb{R}^{n+h}$ are obtained as principal orbits of the tangential representation (at a basic point) of a compact (or non compact dual) symmetric space. A way to obtain these submanifolds explicitly is to consider a real simple noncompact Lie algebra $\mathfrak{g}_{0}$ with Cartan decomposition $\mathfrak{g}_{0}=\mathfrak{k}_{0} \oplus \mathfrak{p}_{0}$. Then $\mathfrak{k}_{0}$ is a maximal compactly embedded subalgebra of $\mathfrak{g}_{0}$ [4, Pr. 7.4, p. 184]. Let $K$ be the analytic subgroup $K$ of $\operatorname{Int}\left(\mathfrak{g}_{0}\right)$ corresponding to the subalgebra $a d_{\mathfrak{g}_{0}}\left(\mathfrak{k}_{0}\right)$ of $a d_{\mathfrak{g}_{0}}\left(\mathfrak{g}_{0}\right)$ which is compact. The principal orbits of the representation of $K$ on $\mathfrak{p}_{0}$ are isoparametric submanifolds $M^{n}$ of $\mathbb{R}^{n+h}=\mathfrak{p}_{0}$. The central objective of this work is to present the following result:

Theorem 1.1. On any compact, connected, homogeneous isoparametric submanifold (for a real simple noncompact Lie algebra $\mathfrak{g}_{0}$ ) there exist a smooth completely non-integrable (i.e., bracket generating) step 2 distribution $\mathfrak{D} \subset T\left(M^{n}\right)$, canonically associated to the manifold.

2010 Mathematics Subject Classification. 53C30, 53C42, 53C17.

Partial support from Universidad Nacional de Córdoba and CONICET, Argentina, is gratefully acknowledged. 
Recall that a distribution $\mathfrak{D}$ of $r$-planes $(n>r \geq 2)$ in a connected manifold $M^{n}$ is smooth [8, p. 41] if for any $p \in M^{n}$ there is an open set $A$ containing $p$ and $r$ smooth vector fields $\left\{X_{1}, \ldots, X_{r}\right\}$ defined on $A$ such that $X_{j}(q) \in \mathfrak{D}(q)$ and $\mathfrak{D}(q)=\operatorname{span}_{\mathbb{R}}\left\{X_{j}(q)\right\}(1 \leq j \leq r, \forall q \in A)$. The distribution $\mathfrak{D}$ is said to be completely non-integrable of step 2 if for every point $p \in M^{n}$ the above vector fields defined in $A$ satisfy $(\forall q \in A)$ :

$$
\operatorname{span}_{\mathbb{R}}\left\{X_{j}(q),\left[X_{k}, X_{j}\right](q): 1 \leq k, j \leq r\right\}=T_{q}(M),
$$

i.e., the generated real vector space coincides with the tangent space.

The presence in $M^{n}$ of the distribution given by Theorem 1.1 has the following consequence. Recall that, given the distribution $\mathfrak{D}$, a curve $\gamma:[0, b] \rightarrow M^{n}$ is said to be horizontal for $\mathfrak{D}$ if $\gamma^{\prime}(t) \in \mathfrak{D}(\gamma(t)) \forall t \in[0, b]$ and regular if $\gamma^{\prime}(t) \neq 0 \forall t$.

Corollary 1.2. Let $M^{n}$ be a compact, connected, homogeneous isoparametric submanifold of $\mathbb{R}^{n+h}$ and consider in $M$ the distribution $\mathfrak{D}$ given by Theorem 1.1 . Then for any two points $p, q$ in $M^{n}$ there exists a horizontal $C^{\infty}$ regular curve $\gamma:[0, b] \rightarrow M^{n}$, such that $\gamma(0)=p, \gamma(b)=q$.

The proof of Theorem 1.1 is naturally divided into two parts by the nature of the system of restricted roots associated to the above Cartan decomposition of the corresponding real simple noncompact Lie algebra $\mathfrak{g}_{0}$. The system of restricted roots can be either the system of roots of a complex simple Lie algebra (reduced case) or $\left(B C_{q}\right)$, in the non-reduced case [4, 3.25, p. 475]. In this first part we take care of the proof of Theorem 1.1 when the system of restricted roots is the system of roots of a complex simple Lie algebra (i.e., it is reduced), and reserve for part 2 of this paper the proof for $\left(B C_{q}\right)$. Nevertheless, in the present part (up to Section 6) we introduce the facts and tools needed for both parts.

Corollary 1.2 is somehow related to one of the results of the important paper 3 . We refer to Theorem D of that paper, which motivated Theorem 1.1. We do not include a proof of Corollary 1.2 since it is a well known consequence of the fact that the distribution $\mathfrak{D}$ is completely non-integrable of step 2.

The rest of the paper contains the description of the distribution $\mathfrak{D}$ and proof of the fact that it is bracket generating of step 2. This is organized as follows. In the next section we collect the necessary facts from Lie theory. The majority of them are taken from the very interesting paper [5], from which we adopt much of the notation and the important Proposition 2.1. The paper [5] has been very useful to help us carry on the somewhat involved computations required. Other more standard facts are recalled from the usual sources (such as [4, 2, 6]). In Section 3 we define our submanifolds and their tangent and normal spaces. Section 4 introduces the basis from [5] and other notations required. In Section 5 we present the distribution and study its local fields and their covariant derivatives and brackets. In Section 6 we indicate some necessary lemmata about properties of the roots of $\mathfrak{g}_{0}^{\mathbb{C}}$ and their restricted counterpart. Finally, Section 7 contains the proof of Theorem 1.1. where the computations of products and brackets performed in the Appendix are extensively used. 


\section{FACTS FROM Lie THEORY}

We shall use some of the notation and a result (Proposition 2.1 below) from the paper [5], which plays an important part here.

Let $\mathfrak{g}$ be a complex simple Lie algebra, $\mathfrak{h} \subset \mathfrak{g}$ a Cartan subalgebra, and $B$ the Killing form of $\mathfrak{g}$. Let $\Phi(\mathfrak{g}, \mathfrak{h}) \subset \mathfrak{h}^{*}$ (dual space) be the root system. Given $\alpha \in$ $\Phi(\mathfrak{g}, \mathfrak{h})$, let $\mathfrak{g}_{\alpha} \subset \mathfrak{g}$ be its root space. Basic properties are in [4, Th. 4.2]; in particular, if $\alpha \neq \pm \beta, \mathfrak{g}_{\alpha}$ is orthogonal to $\mathfrak{g}_{\beta}$ by $B$. Let $t_{\alpha} \in \mathfrak{h}$ be the root vector corresponding to $\alpha$ defined by $B\left(t_{a}, h\right)=\alpha(h), \forall h \in \mathfrak{h}$. Let $\Delta(\mathfrak{g}, \mathfrak{h})=$ $\left\{\alpha_{1}, \ldots, \alpha_{n}\right\} \subset \Phi(\mathfrak{g}, \mathfrak{h})$ be a set of simple roots; we keep $\Delta(\mathfrak{g}, \mathfrak{h})$ and the "order" generated by it in $\Phi(\mathfrak{g}, \mathfrak{h})$, fixed. Set $h_{\alpha}=\frac{2 t_{\alpha}}{B\left(t_{\alpha}, t_{\alpha}\right)}$, and $h_{j}=h_{\alpha_{j}}, 1 \leq j \leq n$. A Chevalley basis of $(\mathfrak{g}, \mathfrak{h})$ is a basis $C=\left\{x_{\alpha}, h_{j}: \alpha \in \Phi(\mathfrak{g}, \mathfrak{h}), 1 \leq j \leq n\right\}$ of $\mathfrak{g}$ with the following properties:

(i) $x_{\alpha} \in \mathfrak{g}_{\alpha}$ and $\left[x_{\alpha}, x_{-\alpha}\right]=-h_{\alpha} \forall \alpha \in \Phi(\mathfrak{g}, \mathfrak{h})$.

(ii) Let $\alpha, \beta \in \Phi(\mathfrak{g}, \mathfrak{h})$ be such that $(\alpha+\beta) \in \Phi(\mathfrak{g}, \mathfrak{h})$. Let $c_{\alpha, \beta} \in \mathbb{C}$ be determined by $\left[x_{\alpha}, x_{\beta}\right]=c_{\alpha, \beta} x_{(\alpha+\beta)}$. Then $c_{\alpha, \beta}=c_{-\alpha,-\beta}$.

The fundamental importance of this basis is that the structure constants are integers. In fact their properties are:

(a) $\left[h_{j}, h_{k}\right]=0$ for $1 \leq j, k \leq n$.

(b) $\left[h_{j}, x_{\alpha}\right]=\alpha\left(h_{j}\right) x_{\alpha}$ for $\alpha \in \Phi(\mathfrak{g}, \mathfrak{h}), 1 \leq j \leq n\left(\Longrightarrow\left[h, x_{\alpha}\right]=\alpha(h) x_{\alpha}\right.$ for $h \in \mathfrak{h})$.

(c) $\left[x_{\alpha}, x_{-\alpha}\right]=-h_{\alpha} \forall \alpha \in \Phi(\mathfrak{g}, \mathfrak{h})$ and $h_{\alpha}$ is a $\mathbb{Z}$ linear combination of $\left\{h_{1}, \ldots, h_{n}\right\}$.

(d) $c_{\alpha, \beta}= \pm(r+1)$, where $r$ is the largest integer such that $\beta-r \alpha \in \Phi(\mathfrak{g}, \mathfrak{h})$.

Let $\mathfrak{g}_{0}$ be a real simple Lie algebra, $\mathfrak{g}_{0}^{\mathbb{C}}$ its complexification, and $\sigma$ the corresponding conjugation with respect to $\mathfrak{g}_{0}$. The Killing forms of both algebras agree on $\mathfrak{g}_{0}$ [4, 6.1, p. 180]; we shall use $B$ for this form in both of them. If $\mathfrak{g}_{0}$ is not complex and one of the algebras $\left(\mathfrak{g}_{0}\right.$ or $\left.\mathfrak{g}_{0}^{\mathbb{C}}\right)$ is simple then so is the other one. A decomposition of $\mathfrak{g}_{0}$ into a direct sum as $\mathfrak{g}_{0}=\mathfrak{k}_{0} \oplus \mathfrak{p}_{0}\left(\mathfrak{k}_{0}\right.$ subalgebra of $\mathfrak{g}_{0}$ and $\mathfrak{p}_{0}$ a subspace) is called a Cartan decomposition of $\mathfrak{g}_{0}$ if there exists a compact real form $\mathfrak{u}_{0}$ of $\mathfrak{g}_{0}^{\mathbb{C}}$ such that the following conditions are satisfied: $\sigma\left(\mathfrak{u}_{0}\right) \subset \mathfrak{u}_{0}$, $\mathfrak{k}_{0}=\mathfrak{g}_{0} \cap \mathfrak{u}_{0}$, and $\mathfrak{p}_{0}=\mathfrak{g}_{0} \cap\left(i \mathfrak{u}_{0}\right)$. Every semisimple Lie algebra $\mathfrak{g}_{0}$ over $\mathbb{R}$ has a Cartan decomposition, unique up to conjugation by an inner automorphism of $\mathfrak{g}_{0}$. Clearly, $\mathfrak{u}_{0}=\mathfrak{k}_{0} \oplus i \mathfrak{p}_{0}$. Let $\tau$ be the conjugation of $\mathfrak{g}_{0}^{\mathbb{C}}=\mathfrak{u}_{0}^{\mathbb{C}}$ with respect to $\mathfrak{u}_{0}$. Then $\sigma$ and $\tau$ commute on $\mathfrak{g}_{0}^{\mathbb{C}}$. Therefore $\theta=\sigma \tau=\tau \sigma$ is an automorphism of $\mathfrak{g}_{0}^{\mathbb{C}}$. Since $\theta$ leaves $\mathfrak{g}_{0}$ invariant, it is an automorphism called in [5] a Cartan involution, and $\mathfrak{k}_{0}$ is a maximal compactly embedded subalgebra of $\mathfrak{g}_{0}$ [4, Pr. 7.4, p. 184].

Let $\mathfrak{g}_{0}$ be a real simple Lie algebra and $\mathfrak{g}_{0}=\mathfrak{k}_{0} \oplus \mathfrak{p}_{0}$ a Cartan decomposition with Cartan involution $\theta$. Let $\mathfrak{h}_{0} \subset \mathfrak{g}_{0}$ be a $\theta$ stable Cartan subalgebra such that $\mathfrak{a}_{0}=\mathfrak{h}_{0} \cap \mathfrak{p}_{0}$ is a subspace of maximal dimension in $\mathfrak{p}_{0}$. Let us consider the complexification $(\mathfrak{g}, \mathfrak{h})$ of $\left(\mathfrak{g}_{0}, \mathfrak{h}_{0}\right)\left(\left(\mathfrak{h}_{0}^{\mathbb{C}}\right)=\mathfrak{h}\right.$ is a Cartan subalgebra of $\left.\left(\mathfrak{g}_{0}^{\mathbb{C}}\right)=\mathfrak{g}\right)$ and, as above, let $\Phi(\mathfrak{g}, \mathfrak{h}) \subset \mathfrak{h}^{*}$ be the root system. We have, of course, $\mathfrak{h}_{0}=\left(\mathfrak{h}_{0} \cap \mathfrak{k}_{0}\right) \oplus\left(\mathfrak{h}_{0} \cap \mathfrak{p}_{0}\right)$ and the roots of $\Phi(\mathfrak{g}, \mathfrak{h})$ take imaginary values on $\left(\mathfrak{h}_{0} \cap \mathfrak{k}_{0}\right)$ 
and real values on $\left(\mathfrak{h}_{0} \cap \mathfrak{p}_{0}\right)$. For a linear functional $\lambda$ in $\mathfrak{a}_{0}^{*}$ it is customary to define

$$
\begin{aligned}
& \mathfrak{g}_{0, \lambda}=\left\{x \in \mathfrak{g}_{0}:[h, x]=\lambda(h) x, \forall h \in \mathfrak{a}_{0}\right\}, \\
& \mathfrak{g}_{0,0}=\left\{x \in \mathfrak{g}_{0}:[h, x]=0, \forall h \in \mathfrak{a}_{0}\right\} .
\end{aligned}
$$

If $\lambda \neq 0$ and $\mathfrak{g}_{0, \lambda} \neq\{0\}$ then $\lambda$ is called a restricted root of $\left(\mathfrak{g}_{0}, \mathfrak{a}_{0}\right)$ and $\mathfrak{g}_{0, \lambda}$ a restricted root space of $\left(\mathfrak{g}_{0}, \mathfrak{a}_{0}\right)$. Let $\Phi\left(\mathfrak{g}_{0}, \mathfrak{a}_{0}\right)$ be the set of restricted roots. $\Phi\left(\mathfrak{g}_{0}, \mathfrak{a}_{0}\right)$ is a system of roots but it may be non-reduced so $\Phi\left(\mathfrak{g}_{0}, \mathfrak{a}_{0}\right)$ is either the system of roots corresponding to a simple complex Lie algebra or the system $(B C)_{q}$ [4, 3.25, p. 475]. Let us denote by $\Phi^{+}\left(\mathfrak{g}_{0}, \mathfrak{a}_{0}\right)$ the set of positive roots for some order in $\Phi\left(\mathfrak{g}_{0}, \mathfrak{a}_{0}\right)$ to be determined below. We have the three involutions $\sigma, \tau$ and $\theta$ defined on the complex simple Lie algebra $\mathfrak{g}=\left(\mathfrak{g}_{0}^{\mathbb{C}}\right)$. They act on $\alpha \in \Phi(\mathfrak{g}, \mathfrak{h})$ as $\alpha^{\sigma}(h)=\overline{\alpha(\sigma(h))}, \alpha^{\tau}(h)=\overline{\alpha(\tau(h))}$, and $\alpha^{\theta}(h)=\alpha(\theta(h))$, for $h \in \mathfrak{h}$. Then $\alpha^{\sigma}, \alpha^{\tau}$, and $\alpha^{\theta}$ are roots of $(\mathfrak{g}, \mathfrak{h})$. For each $\alpha \in \Phi(\mathfrak{g}, \mathfrak{h})$, we have $\alpha^{\tau}=-\alpha([4,3.1$, p. 257], [5]). A root $\alpha \in \Phi(\mathfrak{g}, \mathfrak{h})$ is called real if it takes only real values on $\mathfrak{h}_{0}$, that is, if it vanishes on $\mathfrak{h}_{0} \cap \mathfrak{k}_{0}$. On the other hand $\alpha \in \Phi(\mathfrak{g}, \mathfrak{h})$ is called imaginary if it takes imaginary values on $\mathfrak{h}_{0}$, that is, if it vanishes on $\mathfrak{h}_{0} \cap \mathfrak{p}_{0}=\mathfrak{a}_{0}$. The root is called complex otherwise ([6, p. 390]). Then $\alpha \in \Phi(\mathfrak{g}, \mathfrak{h})$ is imaginary if and only if $\alpha^{\sigma}=-\alpha$ and $\alpha^{\theta}=\alpha$. On the other hand, $\alpha \in \Phi(\mathfrak{g}, \mathfrak{h})$ is real if and only if $\alpha^{\sigma}=\alpha$. We set, as in [5], $\Phi_{\mathbb{R}}$ for the set of real roots, $\Phi_{i \mathbb{R}}$ for the set of imaginary roots, and $\Phi_{\mathbb{C}}$ for the set of complex roots. Furthermore, set $\Sigma=\Phi_{\mathbb{R}} \cup \Phi_{\mathbb{C}}$ and also, recalling that we have the set of simple roots $\Delta(\mathfrak{g}, \mathfrak{h}) \subset \Phi(\mathfrak{g}, \mathfrak{h})$, we also set, as in [5], $\Delta_{0}(\mathfrak{g}, \mathfrak{h})=\Delta(\mathfrak{g}, \mathfrak{h}) \cap \Phi_{i \mathbb{R}}$ and $\Delta_{1}(\mathfrak{g}, \mathfrak{h})=\Delta(\mathfrak{g}, \mathfrak{h}) \cap \Sigma$. We shall need the following proposition ([5, Prop. 3.1]) which will be very important here.

Proposition 2.1. There exists a Chevalley basis

$$
C=\left\{x_{\alpha}, h_{j}: \alpha \in \Phi(\mathfrak{g}, \mathfrak{h}), 1 \leq j \leq n\right\}
$$

of $(\mathfrak{g}, \mathfrak{h})$ such that

(i) $\tau\left(x_{\alpha}\right)=x_{\alpha^{\tau}}=x_{-\alpha}$ for each $\alpha \in \Phi(\mathfrak{g}, \mathfrak{h})$.

(ii) $\sigma\left(x_{\alpha}\right)= \pm x_{\alpha^{\sigma}}$ for each $\alpha \in \Phi(\mathfrak{g}, \mathfrak{h})$ and $\sigma\left(x_{\alpha}\right)=+x_{\alpha^{\sigma}}$ for each $\alpha \in$ $\left(\Phi_{i \mathbb{R}} \cup \Delta_{1}(\mathfrak{g}, \mathfrak{h})\right)$.

We say, with Kammeyer [5], that the Chevalley basis given by this proposition is $\tau$ and $\sigma$ adapted. For the rest of the paper we assume that we have in our simple complex Lie algebra $(\mathfrak{g}, \mathfrak{h})$ a fixed $\tau$ and $\sigma$ adapted Chevalley basis. We use the notation $\rho: \Phi(\mathfrak{g}, \mathfrak{h}) \rightarrow \Phi\left(\mathfrak{g}_{0}, \mathfrak{a}_{0}\right)$ to indicate the restriction of the roots. It is clear that $\rho\left(\Phi_{i \mathbb{R}}\right)=0$. Also, $\alpha^{\sigma}$ and $\alpha$ agree on $\mathfrak{a}_{0}$, so $\rho(\alpha)=\rho\left(\alpha^{\sigma}\right)$ [4, 3.3, p. 260]. Then (see [4, p. 263, p. 408], [5, p. 3]) $\Phi\left(\mathfrak{g}_{0}, \mathfrak{a}_{0}\right)=\rho(\Sigma)$ and $\mathfrak{g}_{0, \lambda}=\left(\sum_{\rho(\alpha)=\lambda} \mathfrak{g}_{\alpha}\right) \cap \mathfrak{g}_{0}$, for $\lambda \in \Phi\left(\mathfrak{g}_{0}, \mathfrak{a}_{0}\right)$.

We have the systems of roots $\Phi(\mathfrak{g}, \mathfrak{h})$ and of restricted roots $\Phi\left(\mathfrak{g}_{0}, \mathfrak{a}_{0}\right)$ and in $\Phi(\mathfrak{g}, \mathfrak{h})$ the simple roots $\Delta(\mathfrak{g}, \mathfrak{h})$ which, in view of the notation indicated above, we may write as: $\Delta(\mathfrak{g}, \mathfrak{h})=\Delta_{0}(\mathfrak{g}, \mathfrak{h}) \cup \Delta_{1}(\mathfrak{g}, \mathfrak{h})$. We may take in $\Phi\left(\mathfrak{g}_{0}, \mathfrak{a}_{0}\right)$ a corresponding system of simple roots $\Delta\left(\mathfrak{g}_{0}, \mathfrak{a}_{0}\right)$ in such a way that $\rho\left(\Delta_{1}(\mathfrak{g}, \mathfrak{h})\right)=$ $\Delta\left(\mathfrak{g}_{0}, \mathfrak{a}_{0}\right)$. Each simple root $\lambda \in \Delta\left(\mathfrak{g}_{0}, \mathfrak{a}_{0}\right)$ is image of either one or two roots of $\Delta_{1}(\mathfrak{g}, \mathfrak{h})$, and those in $\Delta_{0}(\mathfrak{g}, \mathfrak{h})$ go to zero by $\rho$. Fixing in $\Phi(\mathfrak{g}, \mathfrak{h})$ and $\Phi\left(\mathfrak{g}_{0}, \mathfrak{a}_{0}\right)$ the 
orders defined by the respective systems of simple roots $\Delta(\mathfrak{g}, \mathfrak{h})$ and $\Delta\left(\mathfrak{g}_{0}, \mathfrak{a}_{0}\right)$, we clearly have $\rho\left(\Phi^{+}(\mathfrak{g}, \mathfrak{h})\right)=\Phi^{+}\left(\mathfrak{g}_{0}, \mathfrak{a}_{0}\right)$.

For the Killing form $B$ of $\mathfrak{g}_{0}$ we have the associated positive definite, symmetric bilinear form on $\mathfrak{g}_{0}$ defined by

$$
B_{\theta}(x, y):=\langle x, y\rangle_{\theta}=-B(x, \theta y) .
$$

We can write $\mathfrak{g}_{0}$ as an orthogonal (with respect to $B_{\theta}$ ) direct sum of the common eigenspaces of $\left\{a d_{\mathfrak{g}_{0}}(h): h \in \mathfrak{a}\right\}$, that is,

$$
\mathfrak{g}_{0}=\mathfrak{g}_{0,0} \oplus \sum_{\lambda \in \Phi\left(\mathfrak{g}_{0}, \mathfrak{a}_{0}\right)} \mathfrak{g}_{0, \lambda},
$$

and this is usually called the restricted root space decomposition of $\mathfrak{g}_{0}$ with respect to $\mathfrak{a}_{0}$. Since $\theta\left(\mathfrak{g}_{0, \lambda}\right)=\mathfrak{g}_{0,(-\lambda)}\left(\forall \lambda \in \Phi\left(\mathfrak{g}_{0}, \mathfrak{a}_{0}\right)\right)$ and $\theta\left(\mathfrak{g}_{0,0}\right)=\mathfrak{g}_{0,0}$, it follows that $\operatorname{dim} \mathfrak{g}_{0, \lambda}=\operatorname{dim} \mathfrak{g}_{0,(-\lambda)}=m(\lambda) \geq 1$, and $m(\lambda)$ is called the multiplicity of $\lambda$. We also have an orthogonal decomposition $Z_{\mathfrak{g}_{0}}\left(\mathfrak{a}_{0}\right)=\mathfrak{g}_{0,0}=\mathfrak{m}_{0} \oplus \mathfrak{a}_{0}$, where $\mathfrak{m}_{0}=Z_{\mathfrak{k}_{0}}\left(\mathfrak{a}_{0}\right)$ is the centralizer of $\mathfrak{a}_{0}$ in $\mathfrak{k}_{0}$. It is usual to consider also, for $\lambda \in \Phi\left(\mathfrak{g}_{0}, \mathfrak{a}_{0}\right)$, the subspaces

$$
\begin{aligned}
\mathfrak{k}_{0, \lambda} & =\left\{x \in \mathfrak{k}_{0}:(\operatorname{ad}(h))^{2} x=\lambda^{2}(h) x, \forall h \in \mathfrak{a}_{0}\right\}, \\
\mathfrak{p}_{0, \lambda} & =\left\{x \in \mathfrak{p}_{0}:(\operatorname{ad}(h))^{2} x=\lambda^{2}(h) x, \forall h \in \mathfrak{a}_{0}\right\},
\end{aligned}
$$

and obviously $\mathfrak{k}_{0, \lambda}=\mathfrak{k}_{0,(-\lambda)}, \mathfrak{p}_{0, \lambda}=\mathfrak{p}_{0,(-\lambda)}$. We also have orthogonal decompositions with respect to $B_{\theta}$ :

$$
\mathfrak{k}_{0}=\mathfrak{m}_{0} \oplus \sum_{\lambda \in \Phi^{+}\left(\mathfrak{g}_{0}, \mathfrak{a}_{0}\right)} \mathfrak{k}_{0, \lambda}, \quad \mathfrak{p}_{0}=\mathfrak{a}_{0} \oplus \sum_{\lambda \in \Phi^{+}\left(\mathfrak{g}_{0}, \mathfrak{a}_{0}\right)} \mathfrak{p}_{0, \lambda},
$$

and furthermore:

$$
\operatorname{dim}_{\mathbb{R}} \mathfrak{k}_{0, \lambda}=\operatorname{dim}_{\mathbb{R}} \mathfrak{p}_{0, \lambda}=\operatorname{dim}_{\mathbb{R}} \mathfrak{g}_{0, \lambda}=\operatorname{dim}_{\mathbb{R}} \mathfrak{g}_{0,(-\lambda)}=m(\lambda) .
$$

If $E \in \mathfrak{a}_{0}$ is a regular element, we see that $\mathfrak{k}_{0, E}=\left\{x \in \mathfrak{k}_{0}:[x, E]=0\right\}=\mathfrak{m}_{0}$.

\section{The submanifolds}

Let us continue considering our real simple Lie algebra $\mathfrak{g}_{0}$ with Cartan decomposition $\mathfrak{g}_{0}=\mathfrak{k}_{0} \oplus \mathfrak{p}_{0}$ and involution $\theta$. Since $\mathfrak{k}_{0}$ is a maximal compactly embedded subalgebra of $\mathfrak{g}_{0}$, the analytic subgroup $K$ of $\operatorname{Int}\left(\mathfrak{g}_{0}\right)$ corresponding to the subalgebra $a d_{\mathfrak{g}}\left(\mathfrak{k}_{0}\right)$ of $a d_{\mathfrak{g}}\left(\mathfrak{g}_{0}\right)$ is compact. We take in $\mathfrak{g}_{0}$ the inner product (2.1). Then the group $K$ acts on $\left(\mathfrak{p}_{0}, B_{\theta}\right)$ by the adjoint representation (i.e., by isometries) and we consider the principal orbits of this action usually called isoparametric submanifolds. Then we fix a regular element $E \in \mathfrak{a}_{0} \subset \mathfrak{p}_{0}$ and call

$$
M=A d(K) E \subset \mathfrak{p}_{0} ;
$$

also call $K_{E}$ the isotropy subgroup of $K$ at $E$. Since $E$ is regular, the isotropy subalgebra is $\mathfrak{k}_{0, E}=\mathfrak{m}_{0}$. Also by 2.2 the tangent space at $E \in M$ is

$$
T_{E}(M)=\left[\mathfrak{k}_{0}, E\right]=\sum_{\lambda \in \Phi^{+}\left(\mathfrak{g}_{0}, \mathfrak{a}_{0}\right)}\left[\mathfrak{k}_{0, \lambda}, E\right]=\sum_{\lambda \in \Phi^{+}\left(\mathfrak{g}_{0}, \mathfrak{a}_{0}\right)} \mathfrak{p}_{0, \lambda}
$$


and, since both decompositions in 2.2 are orthogonal, the normal space at $E$ is $T_{E}^{\perp}(M)=\mathfrak{a}_{0}$.

It goes without saying that the split (normal) real form $\mathfrak{g}_{S}$ of $\mathfrak{g}$ is included in our considerations. But we need to include also the so called manifolds of complete flags of compact connected simple Lie groups. To that end recall that our compact Lie algebra $\mathfrak{u}_{0}$ is a compact real form of $\mathfrak{g}$, that is, $\mathfrak{u}_{0} \oplus i \mathfrak{u}_{0}=\mathfrak{g}^{\mathbb{R}}$, and this is a Cartan decomposition of the real Lie algebra $\mathfrak{g}^{\mathbb{R}}$ [4, 7.5, p. 185]. Here we may take a compact connected Lie group $G$ corresponding to $\mathfrak{u}_{0}$ (which we may take without center) and consider the principal orbits of $G$ by the adjoint action on the complementary subspace $\left(i \mathfrak{u}_{0}\right)$ or (suppressing the irrelevant factor $i$ ) the adjoint action of $G$ on $\mathfrak{u}_{0}$. In both cases (for $\mathfrak{g}_{S}$ and $\mathfrak{u}_{0}$ ) using Proposition 2.1 one has to make the corresponding "simplifications". For instance $\Phi\left(\mathfrak{g}_{0}, \mathfrak{a}_{0}\right)=\Phi(\mathfrak{g}, \mathfrak{h})$ and $\rho$, $\sigma$ are the identity.

\section{BASIS FOR $\mathfrak{g}_{0}$}

Using part (ii) of Proposition 2.1, we may define $k_{\alpha}$ for each $\alpha \in \Phi(\mathfrak{g}, \mathfrak{h})$ by the identity

$$
\sigma\left(x_{\alpha}\right)=k_{\alpha} x_{\alpha^{\sigma}}, \quad k_{\alpha}= \pm 1
$$

$\sigma\left(x_{\alpha}\right)=k_{\alpha} x_{\alpha^{\sigma}}$ yields $x_{\alpha}=\sigma\left(k_{\alpha} x_{\alpha^{\sigma}}\right)=k_{\alpha} \sigma\left(x_{\alpha^{\sigma}}\right)=k_{\alpha} k_{\alpha^{\sigma}} x_{\alpha}$. Then $k_{\alpha} k_{\alpha^{\sigma}}=1$ and so $k_{\alpha^{\sigma}}=k_{\alpha}$.

Also recalling the effect of $\tau$ ((i) of Proposition 2.1) we may consider the action of $\theta$ on $x_{\alpha}$ and $x_{\alpha^{\sigma}}$, that is,

$$
\begin{gathered}
\theta\left(x_{\alpha}\right)=\tau \sigma\left(x_{\alpha}\right)=\tau\left(k_{\alpha} x_{\alpha^{\sigma}}\right)=k_{\alpha} \tau\left(x_{\alpha^{\sigma}}\right)=k_{\alpha} x_{-\alpha^{\sigma}}, \\
\theta\left(x_{\alpha^{\sigma}}\right)=\tau \sigma\left(x_{\alpha^{\sigma}}\right)=\tau\left(k_{\alpha} x_{\alpha}\right)=k_{\alpha} \tau\left(x_{\alpha}\right)=k_{\alpha} x_{-\alpha} .
\end{gathered}
$$

Now, by the definition (4.1), we have $\sigma\left(x_{-\alpha}\right)=k_{-\alpha} x_{-\alpha^{\sigma}}$ but also

$$
\sigma\left(x_{-\alpha}\right)=\sigma\left(\tau\left(x_{\alpha}\right)\right)=\tau\left(k_{\alpha} x_{\alpha^{\sigma}}\right)=k_{\alpha} \tau\left(x_{\alpha^{\sigma}}\right)=k_{\alpha} x_{-\alpha^{\sigma}},
$$

hence

$$
k_{-\alpha}=k_{\alpha} .
$$

We shall need the basis constructed by Kammeyer in [5, Sec. 4]. Let us consider the $\sigma$ and $\tau$ adapted Chevalley basis for $(\mathfrak{g}, \mathfrak{h})$ from Proposition 2.1 and set, for $\alpha \in \Phi(\mathfrak{g}, \mathfrak{h})$,

$$
X_{\alpha}=x_{\alpha}+\sigma\left(x_{\alpha}\right), \quad Y_{\alpha}=i\left(x_{\alpha}-\sigma\left(x_{\alpha}\right)\right), \quad Z_{\alpha}=X_{\alpha}+Y_{\alpha} .
$$

These vectors are fixed by $\sigma$, so they belong to $\mathfrak{g}_{0}$. Now setting

$$
\begin{array}{lll}
P_{\alpha}=\left(X_{\alpha}+\theta X_{\alpha}\right), & Q_{\alpha}=\left(Y_{\alpha}+\theta Y_{\alpha}\right), & R_{\alpha}=\left(Z_{\alpha}+\theta Z_{\alpha}\right), \\
U_{\alpha}=\left(X_{\alpha}-\theta X_{\alpha}\right), & V_{\alpha}=\left(Y_{\alpha}-\theta Y_{\alpha}\right), & W_{\alpha}=\left(Z_{\alpha}-\theta Z_{\alpha}\right),
\end{array}
$$

we see that the vectors in the first row of (4.4) belong to $\mathfrak{k}_{0}$ and those in the second one to $\mathfrak{p}_{0}$. 
Now, using 4.2 and definitions, we observe that:

$$
\begin{aligned}
& P_{\alpha}=\left(X_{\alpha}+\theta X_{\alpha}\right)=\left(x_{\alpha}+k_{\alpha} x_{\alpha^{\sigma}}\right)+\left(k_{\alpha} x_{-\alpha^{\sigma}}+x_{-\alpha}\right), \\
& U_{\alpha}=\left(X_{\alpha}-\theta X_{\alpha}\right)=\left(x_{\alpha}+k_{\alpha} x_{\alpha^{\sigma}}\right)-\left(k_{\alpha} x_{-\alpha^{\sigma}}+x_{-\alpha}\right), \\
& Q_{\alpha}=\left(Y_{\alpha}+\theta Y_{\alpha}\right)=i\left(x_{\alpha}-k_{\alpha} x_{\alpha^{\sigma}}\right)+i\left(k_{\alpha} x_{-\alpha^{\sigma}}-x_{-\alpha}\right), \\
& V_{\alpha}=\left(Y_{\alpha}-\theta Y_{\alpha}\right)=i\left(x_{\alpha}-k_{\alpha} x_{\alpha^{\sigma}}\right)-i\left(k_{\alpha} x_{-\alpha^{\sigma}}-x_{-\alpha}\right) .
\end{aligned}
$$

On the other hand, the vectors $R_{\alpha}$ and $W_{\alpha}$ shall be considered only for $\alpha$ real (i.e., $\alpha^{\sigma}=\alpha$ ) and clearly $R_{\alpha}=P_{\alpha}+Q_{\alpha}, W_{\alpha}=U_{\alpha}+V_{\alpha}$. However, observe that, for $\alpha$ real, we have the equalities:

$$
\begin{array}{lll}
R_{\alpha}=P_{\alpha}, & W_{\alpha}=U_{\alpha}, & \text { if } k_{\alpha}=1, \\
R_{\alpha}=Q_{\alpha}, & W_{\alpha}=V_{\alpha}, & \text { if } k_{\alpha}=-1 .
\end{array}
$$

Clearly, for $\alpha \in \Phi_{\mathbb{C}}$ and $\beta \in \Phi_{\mathbb{R}}$ we have $P_{\alpha}, Q_{\alpha}, R_{\beta} \in \mathfrak{k}_{0}$ and $U_{\alpha}, V_{\alpha}, W_{\beta} \in \mathfrak{p}_{0}$.

Now, for $h \in \mathfrak{a}_{0}$, (since $\left.\alpha^{\sigma}(h)=\alpha(h)\right)$ we have:

$$
\begin{aligned}
{\left[h, P_{\alpha}\right] } & =\alpha(h)\left(x_{\alpha}-x_{-\alpha}\right)+\alpha^{\sigma}(h) k_{\alpha}\left(x_{\alpha^{\sigma}}-x_{-\alpha^{\sigma}}\right) \\
& =\alpha(h)\left(X_{\alpha}-\theta X_{\alpha}\right)=\alpha(h) U_{\alpha} \\
{\left[h,\left[h, P_{\alpha}\right]\right] } & =\left[h,\left(\alpha(h)\left(\left(x_{\alpha}-x_{-\alpha}\right)+k_{\alpha}\left(x_{\alpha^{\sigma}}-x_{-\alpha^{\sigma}}\right)\right)\right)\right] \\
& =\alpha^{2}(h)\left(X_{\alpha}+\theta X_{\alpha}\right)=\alpha^{2}(h) P_{\alpha},
\end{aligned}
$$

and we conclude that $P_{\alpha}$ is in $\mathfrak{k}_{0 \rho(\alpha)}$. Setting $\rho(\alpha)=\rho(\beta)=\lambda$, similar computations for $\alpha \in \Phi_{\mathbb{C}}, \beta \in \Phi_{\mathbb{R}}$ with the vectors in 4.4 show that

$$
P_{\alpha}, Q_{\alpha}, R_{\beta} \in \mathfrak{k}_{0 \lambda} \quad \text { and } \quad U_{\alpha}, V_{\alpha}, W_{\beta} \in \mathfrak{p}_{0 \lambda}
$$

4.1. Basis for $\mathfrak{k}_{0, \lambda}$ and $\mathfrak{p}_{0, \lambda}, \lambda \in \Phi^{+}\left(\mathfrak{g}_{0}, \mathfrak{a}_{0}\right)$. Consider now for $\lambda \in \Phi^{+}\left(\mathfrak{g}_{0}, \mathfrak{a}_{0}\right)$ the set $\rho^{-1}(\lambda)=\left\{\alpha \in \Phi^{+}(\mathfrak{g}, \mathfrak{h}): \rho(\alpha)=\lambda\right\}$ and split it separating the real roots from the complex ones. So we set $\rho^{-1}(\lambda)_{\mathbb{R}}=\rho^{-1}(\lambda) \cap \Phi_{\mathbb{R}}$ and $\rho^{-1}(\lambda)_{\mathbb{C}}=\rho^{-1}(\lambda) \cap \Phi_{\mathbb{C}}$. For a root $\alpha$ in $\rho^{-1}(\lambda)_{\mathbb{C}}$ we have $\alpha^{\sigma} \neq \alpha$; then we define, as in [5], the set $\rho^{-1}(\lambda)_{\mathbb{C}}^{*}$ where we place one of the two elements in $\left\{\alpha, \alpha^{\sigma}\right\}$ for each $\alpha \in \rho^{-1}(\lambda)_{\mathbb{C}}$. Now for $\lambda, \mu \in \Phi^{+}\left(\mathfrak{g}_{0}, \mathfrak{a}_{0}\right)$ take the sets

$$
\begin{aligned}
& \Xi_{\mathfrak{k}}(\lambda)=\left\{R_{\eta}, P_{\delta}, Q_{\gamma}: \eta \in \rho^{-1}(\lambda)_{\mathbb{R}}, \delta, \gamma \in \rho^{-1}(\lambda)_{\mathbb{C}}^{*}\right\}, \\
& \Xi_{\mathfrak{p}}(\mu)=\left\{W_{\alpha}, U_{\beta}, V_{\varphi}: \alpha \in \rho^{-1}(\mu)_{\mathbb{R}}, \beta, \varphi \in \rho^{-1}(\mu)_{\mathbb{C}}^{*}\right\} .
\end{aligned}
$$

By 4.7), $\Xi_{\mathfrak{k}}(\lambda) \subset \mathfrak{k}_{0 \lambda}$ and $\Xi_{\mathfrak{p}}(\mu) \subset \mathfrak{p}_{0 \mu}$, and each set is linearly independent over $\mathbb{R}$. Since the equal cardinalities of $\Xi_{\mathfrak{k}}(\lambda)$ and $\Xi_{\mathfrak{p}}(\lambda)$ coincide with the dimensions of $\mathfrak{k}_{0, \lambda}$ and $\mathfrak{p}_{0, \lambda}$, we have a basis for each of these subspaces. Obviously, there is a one to one correspondence between $\Xi_{\mathfrak{k}}(\lambda)$ and $\Xi_{\mathfrak{p}}(\lambda)$. As a consequence of (4.6), for the members of the bases $\Xi_{\mathfrak{k}}(\lambda)$ and $\Xi_{\mathfrak{p}}(\lambda)$ we have:

$$
\left[R_{\eta}, E\right]=-\eta(E) W_{\eta}, \quad\left[P_{\delta}, E\right]=-\delta(E) U_{\delta}, \quad\left[Q_{\delta}, E\right]=-\delta(E) V_{\delta},
$$

which is coherent with their one to one correspondence. 


\section{Distribution}

The roots of $\Phi^{+}\left(\mathfrak{g}_{0}, \mathfrak{a}_{0}\right)$ are written in terms of $\Delta\left(\mathfrak{g}_{0}, \mathfrak{a}_{0}\right)$ as a $\mathbb{Z}$ linear combination with non-negative coefficients. It is usual to define the height of a root as the sum of these coefficients, and we may consider in $\Phi^{+}\left(\mathfrak{g}_{0}, \mathfrak{a}_{0}\right)$ the subsets $\Omega$ and $\Gamma$ of roots of odd and even height respectively, $\Phi^{+}\left(\mathfrak{g}_{0}, \mathfrak{a}_{0}\right)=\Omega \cup \Gamma$. We may consider, associated to the set $\Omega$, a subspace $\mathfrak{D}_{E}(\Omega) \subset T_{E}(M)$ (see (3.1)) defined by $\mathfrak{D}_{E}(\Omega)=\sum_{\lambda \in \Omega} \mathfrak{p}_{0 \lambda}$. This subspace is invariant by the action of the isotropy subgroup at $E$. The union of the sets $\Xi_{\mathfrak{p}}(\lambda)$ with $\lambda \in \Omega$ is a basis for $\mathfrak{D}_{E}(\Omega)$. Since $\mathfrak{D}_{E}(\Omega)$ is invariant by $K_{E}$, it defines a distribution $\mathfrak{D}(\Omega)$ on the manifold $M$ by translation with the action of the group $K$. Then at each point $q=A d(g) E \in M$ we have: $\mathfrak{D}_{q}=\mathfrak{D}_{q}(\Omega)=A d(g) \mathfrak{D}_{E}(\Omega) \subset T_{q}(M)$. It is clear that the distribution $\mathfrak{D}(\Omega)$ is well defined. We have to show that it is smooth.

5.1. Smooth local fields. We have in $\mathfrak{g}_{0}$ the inner product $B_{\theta}$ defined in (2.1) (denote its norm by $\|*\|$ ) and in $\mathfrak{k}_{0}$ the orthogonal decomposition (2.2). Set $\mathfrak{n}_{0}=\sum_{\lambda \in \Phi^{+}\left(\mathfrak{g}_{0}, \mathfrak{a}_{0}\right)} \mathfrak{k}_{0, \lambda}$ and take in $\mathfrak{n}_{0}$ an open ball defined by

$$
N(0, r)=\left\{W \in \mathfrak{n}_{0}:\|W\|<r\right\} .
$$

By taking $r>0$ sufficiently small, we may assume that the function $f: N(0, r) \rightarrow M$ defined by $f(W)=A d(\exp W) E$ is a diffeomorphism from $N(0, r)$ onto the open neighborhood $A_{E}=A d(N(0, r)) E$ of $E$ contained in $M$ ([4, pp. 123-124]). Let us consider inside $N(0, r)$ the sphere $\mathbb{S}\left(0, \frac{r}{2}\right)=\left\{W \in N(0, r):\|W\|=\frac{1}{2} r\right\}$.

Let us take now $\lambda \in \Omega$ and consider the basis $\Xi_{\mathfrak{p}}(\lambda)=\left\{W_{\alpha}, U_{\beta}, V_{\beta}\right\}(\rho(\alpha)=$ $\rho(\beta)=\lambda)$ of $\mathfrak{p}_{0, \lambda}$. Let us take for instance $U_{\beta}$. We may define, on some open set contained in $A_{E} \subset M$, a local vector field associated to $U_{\beta}$ by

$$
U_{\beta}^{F}(A d(\exp t L) E):=A d(\exp t L) U_{\beta}, \quad \forall L \in S\left(0, \frac{r}{2}\right), t \in[0,1) .
$$

Proceeding similarly with the vectors $V_{\beta}$ and $W_{\alpha}$, we get the local fields

$$
\left\{U_{\beta}^{F}, V_{\beta}^{F}, W_{\alpha}^{F}: \beta \in \rho^{-1}(\lambda)_{\mathbb{C}}^{*}, \alpha \in \rho^{-1}(\lambda)_{\mathbb{R}}, \lambda \in \Omega\right\} .
$$

They are defined in the open set $N_{E}:=f(N(0, r / 2)) \subset A_{E}$. At the point $E \in M$, they coincide with $\left\{W_{\alpha}, U_{\beta}, V_{\beta}\right\}$ of $\Xi_{\mathfrak{p}}(\lambda)$ and at any other point $q=A d(\exp t L) E \in N_{E}$ they generate $A d(\exp t L) \mathfrak{p}_{0 \lambda} \subset T_{q}(M)$. Since we can define these $m(\lambda)$ fields for each $\lambda \in \Omega$, we have a local basis for $\mathfrak{D}(\Omega)$ in the open set $N_{E}$ containing $E$. It is also clear that these fields are smooth in $N_{E}$.

Now at any other point $p \in M$ there is a $g \in K$ such that $p=A d(g) E$ and we may consider the open set $A d(g) N_{E}$ containing $p$. On such an open set we have a local basis of smooth vector fields defined by translation of those on $N_{E}$ by $A d(g)$. Hence, by the usual definition $([8,1.56$, p. 41$])$, the distribution $\mathfrak{D}(\Omega)$ on $M$ is smooth.

To understand the nature of $\mathfrak{D}(\Omega)$ we compute the brackets of the fields constructed above by using the Levi-Civita connection on $M$ which is torsion free. 
5.2. Covariant derivatives. We use the fact that $M \subset \mathfrak{p}_{0}$ and $\left(\mathfrak{p}_{0}, B_{\theta}\right)$ is a Euclidean space. So we may compute the Euclidean covariant derivative in $\mathfrak{p}_{0}$, which we denote by $\nabla^{E}$, of each field in 5.2 in the direction of each vector of $\Xi_{\mathfrak{p}}(\mu)$ for $\mu \in \Omega$. Since they are all similar, we compute only one of them. Let us take $\gamma \in \rho^{-1}(\lambda), \varphi \in \rho^{-1}(\mu)$ (we may have $\lambda=\mu$ but in that case $\gamma \neq \varphi$ ).

To compute $\nabla_{U_{\varphi}}^{E} U_{\gamma}^{F}$, we need to know the field $U_{\gamma}^{F}$ restricted to a curve whose tangent vector at $E$ is $U_{\varphi}$, and to obtain it let us consider first the curve on $M$ passing through $E$ defined on an adequate interval $(-\varepsilon, \varepsilon) \subset \mathbb{R}$ by $u(t)=$ $A d\left(\exp \left(t\left(F P_{\varphi}\right)\right)\right) E$, for $t \in(-\varepsilon, \varepsilon)$. Here $F$ is the factor needed so that $F P_{\varphi} \in$ $\mathbb{S}\left(0, \frac{r}{2}\right)$; it will be irrelevant at the end so we keep it as a non-zero undefined constant associated to $P_{\varphi}$. By 4.9 we have

$$
\left.\frac{d}{d t}\right|_{t=0} u(t)=\left[F P_{\varphi}, E\right]=F\left[P_{\varphi}, E\right]=-F \varphi(E) U_{\varphi}
$$

Then (since $E$ is regular and $\varphi \in \rho^{-1}(\mu), \varphi(E)=\mu(E)$ ) we may write

$$
U_{\varphi}=\left.\left(\frac{-1}{F \mu(E)}\right) \frac{d}{d t}\right|_{t=0} A d\left(\exp \left(t F P_{\varphi}\right)\right) E .
$$

So $U_{\varphi}$ is the tangent vector (at $t=0$, i.e., at $E$ ) to the curve in $M$ defined on $(-\varepsilon, \varepsilon) \subset \mathbb{R}$ by

$$
\omega(t)=\left(\frac{-1}{F \mu(E)}\right) A d\left(\exp \left(t F P_{\varphi}\right)\right) E=A d\left(\exp \left(t F P_{\varphi}\right)\right)\left(\frac{-1}{F \mu(E)}\right) E .
$$

Now we need the restriction of the field $U_{\gamma}^{F}$ to the curve $\omega(t)$. By the definition (5.1) we see that this restriction $U_{\gamma}^{F}(\omega(t))$ is

$$
U_{\gamma}^{F}(\omega(t))=\left(\frac{-1}{F \mu(E)}\right) A d\left(\exp \left(t F P_{\varphi}\right)\right) U_{\gamma} .
$$

Then we may compute

and get

$$
\nabla_{U_{\varphi}}^{E} U_{\gamma}^{F}=\left.\frac{d}{d t}\right|_{t=0} U_{\gamma}^{F}(\omega(t))=\left.\left(\frac{-1}{F \mu(E)}\right) \frac{d}{d t}\right|_{t=0} \operatorname{Ad}\left(\exp \left(t F P_{\varphi}\right)\right) U_{\gamma}
$$

$$
\nabla_{U_{\varphi}}^{E} U_{\gamma}^{F}=\left(\frac{-1}{\mu(E)}\right)\left[P_{\varphi}, U_{\gamma}\right]
$$

Now we may obtain $\nabla_{U_{\varphi}} U_{\gamma}^{F}$ by taking the tangential component of $\nabla_{U_{\varphi}}^{E} U_{\gamma}^{F}$. So we have:

$$
\nabla_{U_{\varphi}} U_{\gamma}^{F}=\left(\frac{-1}{\mu(E)}\right) T a\left(\left[P_{\varphi}, U_{\gamma}\right]\right) .
$$

5.3. Brackets. The bracket of the fields $U_{\varphi}^{F}$ and $U_{\gamma}^{F}$ at $E$ is now

$$
\left[U_{\varphi}^{F}, U_{\gamma}^{F}\right](E)=\nabla_{U_{\varphi}} U_{\gamma}^{F}-\nabla_{U_{\gamma}} U_{\varphi}^{F}
$$

and using (5.3), since $\gamma \in \rho^{-1}(\lambda)$ and $\varphi \in \rho^{-1}(\mu)$, we have

$$
\left[U_{\varphi}^{F}, U_{\gamma}^{F}\right](E)=\left(\frac{-1}{\mu(E)}\right) T a\left(\left[P_{\varphi}, U_{\gamma}\right]\right)-\left(\frac{-1}{\lambda(E)}\right) T a\left(\left[P_{\gamma}, U_{\varphi}\right]\right) .
$$


In (5.4) we have brackets of fields on the left side and products in $\mathfrak{g}_{0}$ on the right side. We use the words brackets for fields and products for vectors in $\mathfrak{g}_{0}$. Recall that for $\lambda, \mu \in \Omega$, we have bases $\Xi_{\mathfrak{p}}(\lambda)$ for $\mathfrak{p}_{0 \lambda}$ and $\Xi_{\mathfrak{p}}(\mu)$ for $\mathfrak{p}_{0 \mu}$, respectively. To fix notation we set them as

$$
\begin{aligned}
& \Xi_{\mathfrak{p}}(\lambda)=\left\{U_{\gamma}, V_{\gamma}, W_{\delta}: \gamma \in \rho^{-1}(\lambda)_{\mathbb{C}}^{*}, \delta \in \rho^{-1}(\lambda)_{\mathbb{R}}\right\}, \\
& \Xi_{\mathfrak{p}}(\mu)=\left\{U_{\varphi}, V_{\varphi}, W_{\eta}: \varphi \in \rho^{-1}(\mu)_{\mathbb{C}}^{*}, \eta \in \rho^{-1}(\mu)_{\mathbb{R}}\right\} .
\end{aligned}
$$

Each of these tangent vectors at $E$ generates a corresponding field around $E$ $\left\{U_{\gamma}^{F}, V_{\gamma}^{F}, W_{\beta}^{F}\right\}$ and $\left\{U_{\varphi}^{F}, V_{\varphi}^{F}, W_{\delta}^{F}\right\}$. So we have nine possible brackets of these fields.

\section{Some ReQuired Lemmata}

It is convenient to introduce the following notation. For a root $\alpha$ contained either in $\Phi(\mathfrak{g}, \mathfrak{h})$ or in $\Phi\left(\mathfrak{g}_{0}, \mathfrak{a}_{0}\right)$ we shall write

$$
|\alpha|= \begin{cases}\alpha, & \text { if } \alpha \in \Phi^{+}(\mathfrak{g}, \mathfrak{h})\left(\text { resp. } \Phi^{+}\left(\mathfrak{g}_{0}, \mathfrak{a}_{0}\right)\right) \\ -\alpha, & \text { if }-\alpha \in \Phi^{+}(\mathfrak{g}, \mathfrak{h})\left(\text { resp. } \Phi^{+}\left(\mathfrak{g}_{0}, \mathfrak{a}_{0}\right)\right) .\end{cases}
$$

Recall that $\Delta\left(\mathfrak{g}_{0}, \mathfrak{a}_{0}\right) \subset \Phi^{+}\left(\mathfrak{g}_{0}, \mathfrak{a}_{0}\right)$ is a system of simple roots for $\Phi\left(\mathfrak{g}_{0}, \mathfrak{a}_{0}\right)$. In this Part 1 , we are assuming that $\Phi\left(\mathfrak{g}_{0}, \mathfrak{a}_{0}\right)$ is the system of roots of a complex simple Lie algebra (i.e., it is reduced).

In the following Lemma 6.1 we assume that $\mathfrak{g}_{0} \neq \mathfrak{g}_{2}$, the real form of $\mathfrak{g}_{2}^{\mathbb{C}}$. The case of $\mathfrak{g}_{2}$ is considered in Lemma 6.2 below.

Lemma 6.1. For $\mathfrak{g}_{0} \neq \mathfrak{g}_{2}$, given $\gamma \in \Gamma \subset \Phi^{+}\left(\mathfrak{g}_{0}, \mathfrak{a}_{0}\right)$, we can find $\eta$ and $\delta$ in $\Omega \subset \Phi^{+}\left(\mathfrak{g}_{0}, \mathfrak{a}_{0}\right)$ such that $\eta \neq \delta, \gamma=\eta+\delta$, and $|\eta-\delta|$ is not a root of $\Phi^{+}\left(\mathfrak{g}_{0}, \mathfrak{a}_{0}\right)$.

Proof. This lemma is obtained by inspection of the table of roots in [2, pp.528531]. The mentioned table contains the form of the positive roots for the four types of classical algebras and the five exceptional ones. In the case of the classical algebras, if we take $\gamma \in \Gamma$, it must have an even number of coefficients 1 , therefore it must contain a coefficient 1 at the left of the first obligatory filling $\underline{\mathbf{1}}$ (first from the left, underlined in the table in [2]). Therefore, eliminating from $\gamma$ the root corresponding to the coefficient 1 at the extreme left position (corresponding to some $\left.\alpha_{j} \in \Delta\left(\mathfrak{g}_{0}, \mathfrak{a}_{0}\right)\right)$ we obtain a $\operatorname{root} \beta$ in $\Omega$. Then we may write $\gamma=\beta+\alpha_{j}$ and clearly $\left|\beta-\alpha_{j}\right|$ is not a root of $\Phi\left(\mathfrak{g}_{0}, \mathfrak{a}_{0}\right)$.

On the other hand, for the four exceptional algebras $\mathfrak{e}_{6}^{\mathbb{C}}, \mathfrak{e}_{7}^{\mathbb{C}}, \mathfrak{e}_{8}^{\mathbb{C}}$ and $\mathfrak{f}_{4}^{\mathbb{C}}$ the tables are ordered by increasing height (altitudes in [2]) so the roots of $\Gamma$, in each case, are the ones contained in the rows in even position from the top while those in $\Omega$ are in the other rows. The roots in the $2 k$-th row are constructed from those in the $(2 k-1)$-th row by adding one of the simple roots in the first row. Then we see that any $\gamma \in \Gamma$ can be written as $\gamma=\eta+\delta$, with $\eta, \delta \in \Omega$ and $|\delta-\eta|$ is not a root. 
Lemma 6.2. For $\mathfrak{g}_{2}$, given $\gamma \in \Gamma \subset \Phi^{+}\left(\mathfrak{g}_{2}, \mathfrak{a}\right)$, we can find $\eta \neq \delta$ in $\Omega \subset \Phi^{+}\left(\mathfrak{g}_{0}, \mathfrak{a}_{0}\right)$ such that

$$
\begin{aligned}
\text { either } \gamma & =\eta+\delta \text { and }|\eta-\delta| \text { is not a root of } \Phi^{+}\left(\mathfrak{g}_{0}, \mathfrak{a}_{0}\right) \\
\text { or } \gamma & =|\eta-\delta| \text { and } \eta+\delta \text { is not a root of } \Phi^{+}\left(\mathfrak{g}_{0}, \mathfrak{a}_{0}\right)
\end{aligned}
$$

Proof. Let us take $\mathfrak{g}_{2}^{\mathbb{C}}\left[\begin{array}{ccc}2 & 3 \\ 0 & \Rightarrow & 0 \\ \alpha_{1} & & \alpha_{2}\end{array}\right]$ ( $\alpha_{2}$ is short). The positive roots are

$$
\left\{\alpha_{1}, \alpha_{2},\left(\alpha_{1}+\alpha_{2}\right),\left(\alpha_{1}+2 \alpha_{2}\right),\left(\alpha_{1}+3 \alpha_{2}\right),\left(2 \alpha_{1}+3 \alpha_{2}\right)\right\}
$$

and $\Gamma=\left\{\left(\alpha_{1}+\alpha_{2}\right),\left(\alpha_{1}+3 \alpha_{2}\right)\right\}$, while the other roots are in $\Omega$. We may write $\left(\alpha_{1}+\alpha_{2}\right)=\alpha_{1}+\alpha_{2}$ and $\left(\alpha_{1}-\alpha_{2}\right)$ is not a root. Now

$$
\gamma=\left(\alpha_{1}+3 \alpha_{2}\right)=\left(\alpha_{1}+2 \alpha_{2}\right)+\alpha_{2},
$$

but

$$
\left|\left(\alpha_{1}+2 \alpha_{2}\right)-\alpha_{2}\right|=\left(\alpha_{1}+\alpha_{2}\right) \text { is a root, }
$$

and there is no other way to write $\gamma$ as a sum of two roots in $\Omega$. However, we may write

$$
\left(\alpha_{1}+3 \alpha_{2}\right)=\left|\left(2 \alpha_{1}+3 \alpha_{2}\right)-\alpha_{1}\right|
$$

and

$$
\left(2 \alpha_{1}+3 \alpha_{2}\right)+\alpha_{1}=3 \alpha_{1}+3 \alpha_{2} \text { is not a root. }
$$

This completes the proof.

For the rest of the present section we assume that $\mathfrak{g}_{0} \neq \mathfrak{g}_{2}$.

Lemma 6.3. Given $\lambda \in \Gamma \subset \Phi^{+}\left(\mathfrak{g}_{0}, \mathfrak{a}_{0}\right)$, by Lemma 6.1 there exist two roots $\eta \neq \delta$ in $\Omega \subset \Phi^{+}\left(\mathfrak{g}_{0}, \mathfrak{a}_{0}\right)$ such that $\lambda=\eta+\delta$ and $|\eta-\delta|$ is not a root of $\Phi^{+}\left(\mathfrak{g}_{0}, \mathfrak{a}_{0}\right)$. Then for any root $\gamma \in \rho^{-1}(\lambda) \subset \Phi^{+}(\mathfrak{g}, \mathfrak{h})$ there exist roots $\alpha \in \rho^{-1}(\eta)$ and $\beta \in \rho^{-1}(\delta)$ such that $\gamma=\alpha+\beta$.

Proof. This is proved by inspection in the pairs $\left(\Phi^{+}(\mathfrak{g}, \mathfrak{h}), \Phi^{+}\left(\mathfrak{g}_{0}, \mathfrak{a}_{0}\right)\right)$. The table indicating the pairs $\left(\Phi^{+}(\mathfrak{g}, \mathfrak{h}), \Phi^{+}\left(\mathfrak{g}_{0}, \mathfrak{a}_{0}\right)\right)$ is in [4, pp. 532-534]; the ones to be considered are those where all $m(2 \lambda)=0$.

\section{Proof of Theorem 1.1}

Here we use the results in Subsection 8.5.1 of the Appendix.

Let us observe that in order to prove Theorem 1.1 it is enough to show that, for each $\lambda \in \Gamma$, each vector of the basis $\Xi_{\mathfrak{p}}(\lambda)$ of $\mathfrak{p}_{0 \lambda} \subset T_{E}(M)$ may be computed as a sum of brackets of local fields (defined around $E$ ) that belong to the distribution $\mathfrak{D}(\Omega)$. Let us take then $\lambda \in \Gamma$ and recall the basis of $\mathfrak{p}_{0 \lambda}$ given in 4.8 .

We start considering $\gamma \in \rho^{-1}(\lambda)_{\mathbb{C}}^{*}$ for $\lambda \in \Gamma \subset \Phi^{+}\left(\mathfrak{g}_{0}, \mathfrak{a}_{0}\right)$ and take $U_{\gamma}, V_{\gamma}$ for our chosen $\gamma$. By Lemma 6.1. there exist two roots $\eta$ and $\delta$ in $\Omega \subset \Phi^{+}\left(\mathfrak{g}_{0}, \mathfrak{a}_{0}\right)$ such that

$$
\eta \neq \delta, \quad \lambda=\eta+\delta, \quad|\eta-\delta| \text { is not a root of } \Phi^{+}\left(\mathfrak{g}_{0}, \mathfrak{a}_{0}\right) .
$$


Furthermore, by Lemma 6.3. for the root $\gamma \in \rho^{-1}(\lambda)$ there exist roots $\alpha \in$ $\rho^{-1}(\eta)$ and $\beta \in \rho^{-1}(\delta)$ such that $\gamma=\alpha+\beta$. Then we have $U_{\gamma}=U_{(\alpha+\beta)}$, $V_{\gamma}=V_{(\alpha+\beta)}$, and by 8.11) in the Appendix we have:

$$
\begin{gathered}
\Theta_{(\eta, \delta, \alpha, \beta)} U_{(\alpha+\beta)}+\Lambda_{(\eta, \delta)} T a\left(H_{1}\right)=\left[U_{\alpha}^{F}, U_{\beta}^{F}\right](E)-\left[V_{\alpha}^{F}, V_{\beta}^{F}\right](E), \\
\Theta_{(\eta, \delta, \alpha, \beta)} V_{(\alpha+\beta)}+\Lambda_{(\eta, \delta)} T a\left(T_{2}\right)=\left[U_{\alpha}^{F}, V_{\beta}^{F}\right](E)+\left[V_{\alpha}^{F}, U_{\beta}^{F}\right](E) .
\end{gathered}
$$

Let us study now the terms $H_{1}(8.4)$ and $T_{2}(8.6)$. For the pair of roots $(\alpha, \beta)$, they are:

$$
\begin{aligned}
H_{1} & =2 k_{\alpha} c_{\alpha^{\sigma},-\beta}\left(x_{-\alpha^{\sigma}+\beta}-x_{\alpha^{\sigma}-\beta}\right)-2 k_{\beta} c_{\alpha,-\beta^{\sigma}}\left(x_{\alpha-\beta^{\sigma}}-x_{-\alpha+\beta^{\sigma}}\right), \\
T_{2} & =2 i k_{\alpha} c_{\alpha^{\sigma},-\beta}\left(x_{\alpha^{\sigma}-\beta}+x_{-\alpha^{\sigma}+\beta}\right)-2 i k_{\beta} c_{\alpha,-\beta^{\sigma}}\left(x_{\alpha-\beta^{\sigma}}+x_{-\alpha+\beta^{\sigma}}\right) .
\end{aligned}
$$

In our present situation we have that

$$
\left|\beta-\alpha^{\sigma}\right| \text { and }\left|\alpha-\beta^{\sigma}\right| \text { are not roots of } \Phi(\mathfrak{g}, \mathfrak{h}) \text {. }
$$

In fact we have that $\eta$ and $\delta$ satisfy (7.1) and $\alpha \in \rho^{-1}(\eta), \beta \in \rho^{-1}(\delta)$. If $\left|\beta-\alpha^{\sigma}\right|$ were a root of $\Phi(\mathfrak{g}, \mathfrak{h})$ then $\rho\left(\left|\beta-\alpha^{\sigma}\right|\right)=|\eta-\delta|$ would be a root of $\Phi^{+}\left(\mathfrak{g}_{0}, \mathfrak{a}_{0}\right)$, which is not the case by 7.1$)$. Similarly $\left|\alpha-\beta^{\sigma}\right|$ is not a root of $\Phi(\mathfrak{g}, \mathfrak{h})$. This clearly yields that $H_{1}=T_{2}=0$, and going back to $\sqrt{7.2}$ we see that $U_{\gamma}=U_{(\alpha+\beta)}$, $V_{\gamma}=V_{(\alpha+\beta)}$ are sums of brackets (evaluated on $E$ ) of local fields defined around $E$ that belong to the distribution $D(\Omega)$.

It remains to consider the case of real roots. Let us take so $\varphi \in \rho^{-1}(\lambda)_{\mathbb{R}}$ for $\lambda \in \Gamma \subset \Phi^{+}\left(\mathfrak{g}_{0}, \mathfrak{a}_{0}\right)$; then we have the vector $W_{\varphi}$. Again there exist two roots $\eta$ and $\delta$ in $\Omega \subset \Phi^{+}\left(\mathfrak{g}_{0}, \mathfrak{a}_{0}\right)$ satisfying 7.1 and roots $\xi \in \rho^{-1}(\eta), \omega \in \rho^{-1}(\delta)$ such that $\varphi=\xi+\omega$. Then we have the following possibilities:

(i) $\xi$ and $\omega$ are both real roots of $\Phi(\mathfrak{g}, \mathfrak{h})$;

(ii) $\xi$ and $\omega$ are both complex roots of $\Phi(\mathfrak{g}, \mathfrak{h})$.

In fact, clearly we cannot have that one of them is real and the other complex. But they can be both complex and in that case we also have $\varphi=\xi^{\sigma}+\omega^{\sigma}$. Since $\xi \in \rho^{-1}(\eta), \omega \in \rho^{-1}(\delta)$, we have $\xi^{\sigma} \in \rho^{-1}(\eta), \omega^{\sigma} \in \rho^{-1}(\delta)$, and $\varphi=\xi^{\sigma}+\omega^{\sigma}$ is another decomposition of $\varphi$.

Considering first the case (i) in 7.4 we see, by the argument above, that 7.3 holds in this case and it takes the form

$$
(\xi-\omega) \text { and }(\omega-\xi) \text { are not roots of } \Phi(\mathfrak{g}, \mathfrak{h}) .
$$

Now considering formulae 8.13 (for $\lambda=\eta, \mu=\delta, \xi=\delta, \beta=\omega$, and $\varphi=\xi+\omega$ ) we see, for the pair of roots $(\xi, \omega)$, that formulae (8.4) and (8.6) yield $H_{1}=T_{2}=0$ and therefore the vector $W_{\varphi}$ is a bracket (evaluated at $E$ ) of local fields defined around $E$ that belong to the distribution $D(\Omega)$. On the other hand, in case (ii) of (7.4), we have that 7.3 holds for the pair of complex roots $(\xi, \omega)$, which again yields $H_{1}=T_{2}=0$. Then by formulae (8.12) (for $\lambda=\eta, \mu=\delta, \xi=\delta, \beta=\omega$, and $\varphi=\xi+\omega)$ again in this case $W_{\varphi}$ is a sum of brackets (evaluated at $E$ ) of local fields that belong to the distribution $D(\Omega)$.

This completes the proof of Theorem 1.1 when $\Phi\left(\mathfrak{g}_{0}, \mathfrak{a}_{0}\right)$ is reduced. 
7.1. Comment on the split cases. Since in the split cases $\Phi^{+}(\mathfrak{g}, \mathfrak{h})=\Phi^{+}\left(\mathfrak{g}_{0}, \mathfrak{a}_{0}\right)$ and $\rho$ and $\sigma$ are the identity, Lemma 6.1 takes care of this case. In fact all roots are real and Lemma 6.1 indicates that given $\gamma \in \Gamma \subset \Phi^{+}\left(\mathfrak{g}_{0}, \mathfrak{a}_{0}\right)=\Phi^{+}(\mathfrak{g}, \mathfrak{h})$, we can find $\eta$ and $\delta$ in $\Omega \subset \Phi^{+}\left(\mathfrak{g}_{0}, \mathfrak{a}_{0}\right)$ such that $\eta \neq \delta$ and they satisfy $\gamma=\eta+\delta$ and $|\eta-\delta|$ is not a root of $\Phi^{+}\left(\mathfrak{g}_{0}, \mathfrak{a}_{0}\right)$. Considering formulae 8.4 and 8.6) (for the roots $\eta$ and $\delta$ ) we have (since $\eta-\delta$ and $\delta-\eta$ are not roots) that $H_{1}=T_{2}=0$. Then we may consider formulae (8.13), for which we may write a shortened version:

$$
\Theta W_{\gamma}=\Theta W_{(\eta+\delta)}=( \pm)\left[W_{\eta}^{F}, W_{\delta}^{F}\right](E), \quad \Theta \neq 0,
$$

and therefore the vector $W_{\gamma}$ is a bracket (evaluated at $E$ ) of local fields defined around $E$ that belong to the distribution $D(\Omega)$. This proves the theorem for the split cases.

\section{APPENDIX}

8.1. Basic identities. We need to mention some basic identities that are used in the required computations. Since $\sigma\left(x_{(\alpha+\beta)}\right)=k_{(\alpha+\beta)} x_{(\alpha+\beta)^{\sigma}}$ and $\left[x_{\alpha}, x_{\beta}\right]=$ $c_{\alpha, \beta} x_{(\alpha+\beta)}$ with real coefficients $c_{\alpha, \beta}$, we have

$$
\begin{aligned}
& \sigma\left[x_{\alpha}, x_{\beta}\right]=\sigma\left(c_{\alpha, \beta} x_{\alpha+\beta}\right)=c_{\alpha, \beta} \sigma\left(x_{\alpha+\beta}\right)=c_{\alpha, \beta} k_{(\alpha+\beta)} x_{(\alpha+\beta)^{\sigma}}, \\
& \sigma\left[x_{\alpha}, x_{\beta}\right]=\left[\sigma x_{\alpha}, \sigma x_{\beta}\right]=\left[k_{\alpha} x_{\alpha^{\sigma}}, k_{\beta} x_{\beta^{\sigma}}\right]=k_{\alpha} k_{\beta} c_{\alpha^{\sigma}, \beta^{\sigma}} x_{\left(\alpha^{\sigma}+\beta^{\sigma}\right)} .
\end{aligned}
$$

By repeating this computation for $\left[x_{\alpha}, x_{-\beta}\right],\left[x_{-\alpha}, x_{\beta}\right]$, and $\left[x_{-\alpha}, x_{-\beta}\right]$ (using $\left(c_{-\alpha,-\beta}=c_{\alpha, \beta}\right)$ and 4.3$)$ we get the four identities

$$
\begin{aligned}
k_{\alpha} k_{\beta} c_{\alpha^{\sigma}, \beta^{\sigma}} x_{\alpha^{\sigma}+\beta^{\sigma}} & =c_{\alpha, \beta} k_{(\alpha+\beta)} x_{(\alpha+\beta)^{\sigma}}, \\
k_{\alpha} k_{\beta} c_{\alpha^{\sigma},-\beta^{\sigma}} x_{\alpha^{\sigma}-\beta^{\sigma}} & =c_{\alpha,-\beta} k_{(\alpha-\beta)} x_{(\alpha-\beta)^{\sigma}}, \\
k_{\alpha} k_{\beta} c_{-\alpha^{\sigma}, \beta} x_{-\alpha^{\sigma}+\beta^{\sigma}} & =c_{-\alpha, \beta} k_{(-\alpha+\beta)} x_{(-\alpha+\beta)^{\sigma}}, \\
k_{\alpha} k_{\beta} c_{-\alpha^{\sigma},-\beta^{\sigma}} x_{-\alpha^{\sigma}-\beta^{\sigma}} & =c_{\alpha, \beta} k_{(\alpha+\beta)} x_{-(\alpha+\beta)^{\sigma}} .
\end{aligned}
$$

8.2. Products. By (5.4), to get the brackets of tangent fields we take the bases $\Xi_{\mathfrak{k}}(\lambda)$ and $\Xi_{\mathfrak{p}}(\mu)$ in $(4.8)$, for $\mathfrak{k}_{0, \lambda}$ and $\mathfrak{p}_{0, \mu}$ respectively. There are nine possible products, namely:
(1) $\left[R_{\eta}, W_{\alpha}\right]$,
(2) $\left[R_{\eta}, U_{\beta}\right]$,
(3) $\left[R_{\eta}, V_{\varphi}\right]$,
(4) $\left[P_{\delta}, W_{\alpha}\right]$,
(5) $\left[P_{\delta}, U_{\beta}\right]$,
(6) $\left[P_{\delta}, V_{\varphi}\right]$,
(7) $\left[Q_{\gamma}, W_{\alpha}\right]$
(8) $\left[Q_{\gamma}, U_{\beta}\right]$,
(9) $\left[Q_{\gamma}, V_{\varphi}\right]$.

However, we shall need only (1) (for $\eta, \alpha$ real) and (5), (6), (8), and (9) for complex roots. Now we compute the products $(5),(6),(8)$, and (9) in 8.2 . For reasons of space, we will not perform all these computations explicitly. We take $(5)\left[P_{\delta}, U_{\beta}\right]$ and compute the product:

$$
\begin{aligned}
P_{\delta} & =\left(x_{\delta}+k_{\delta} x_{\delta^{\sigma}}\right)+\left(k_{\delta} x_{-\delta^{\sigma}}+x_{-\delta}\right), \\
U_{\beta} & =\left(x_{\beta}+k_{\beta} x_{\beta^{\sigma}}\right)-\left(k_{\beta} x_{-\beta^{\sigma}}+x_{-\beta}\right),
\end{aligned}
$$


(5) $\left[P_{\delta}, U_{\beta}\right]=c_{\delta, \beta} x_{\delta+\beta}+k_{\beta} c_{\delta, \beta^{\sigma}} x_{\delta+\beta^{\sigma}}+k_{\delta} c_{\delta^{\sigma}, \beta} x_{\delta^{\sigma}+\beta}+k_{\delta} k_{\beta} c_{\delta^{\sigma}, \beta^{\sigma}} x_{\delta^{\sigma}+\beta^{\sigma}}$

$$
\begin{aligned}
& -k_{\beta} c_{\delta,-\beta^{\sigma}} x_{\delta-\beta^{\sigma}}-c_{\delta,-\beta} x_{\delta-\beta}-k_{\delta} k_{\beta} c_{\delta^{\sigma},-\beta^{\sigma}} x_{\delta^{\sigma}-\beta^{\sigma}}-k_{\delta} c_{\delta^{\sigma},-\beta} x_{\delta^{\sigma}-\beta} \\
& +k_{\delta} c_{-\delta^{\sigma}, \beta} x_{-\delta^{\sigma}+\beta}+k_{\delta} k_{\beta} c_{-\delta^{\sigma}, \beta^{\sigma}} x_{-\delta^{\sigma}+\beta^{\sigma}}+c_{-\delta, \beta} x_{-\delta+\beta} \\
& +k_{\beta} c_{-\delta, \beta^{\sigma}} x_{-\delta+\beta^{\sigma}}-k_{\delta} k_{\beta} c_{-\delta^{\sigma},-\beta^{\sigma}} x_{-\delta^{\sigma}-\beta^{\sigma}}-k_{\delta} c_{-\delta^{\sigma},-\beta} x_{-\delta^{\sigma}-\beta} \\
& -k_{\beta} c_{-\delta,-\beta^{\sigma}} x_{-\delta-\beta^{\sigma}}-c_{-\delta,-\beta} x_{-\delta-\beta} .
\end{aligned}
$$

There are four terms with $k_{\delta} k_{\beta}$ which can be replaced using the above identities 8.1. Now we observe that

$$
\begin{aligned}
c_{\delta, \beta} U_{(\delta+\beta)} & =c_{\delta, \beta}\left(x_{(\delta+\beta)}+k_{(\delta+\beta)} x_{(\delta+\beta)^{\sigma}}-k_{(\delta+\beta)} x_{-(\delta+\beta)^{\sigma}}-x_{-(\delta+\beta)}\right), \\
(-1) c_{\delta,-\beta} U_{(\delta-\beta)} & =-c_{\delta,-\beta}\left(x_{(\delta-\beta)}+k_{(\delta-\beta)} x_{(\delta-\beta)^{\sigma}}-k_{(\delta-\beta)} x_{-(\delta-\beta)^{\sigma}}-x_{-(\delta-\beta)}\right),
\end{aligned}
$$

and we see that these eight terms are present in the product (5), so we may replace them in the previous expressions (recalling that $c_{\delta, \beta}=c_{-\delta,-\beta}$ and $k_{(\delta-\beta)}=k_{(\beta-\delta)}$ ). Then the product (5) can be written as

$$
\begin{aligned}
{\left[P_{\delta},\right.} & \left.U_{\beta}\right] \\
= & c_{\delta, \beta} U_{(\delta+\beta)} \\
& +k_{\beta} c_{\delta, \beta^{\sigma}} x_{\delta+\beta^{\sigma}}+k_{\delta} c_{\delta^{\sigma}, \beta} x_{\delta^{\sigma}+\beta}-k_{\delta} c_{-\delta^{\sigma},-\beta} x_{-\delta^{\sigma}-\beta}-k_{\beta} c_{-\delta,-\beta^{\sigma}} x_{-\delta-\beta^{\sigma}} \\
& +(-1) c_{\delta,-\beta} U_{(\delta-\beta)} \\
& -k_{\delta} c_{\delta^{\sigma},-\beta} x_{\delta^{\sigma}-\beta}+k_{\delta} c_{-\delta^{\sigma}, \beta} x_{-\delta^{\sigma}+\beta}-k_{\beta} c_{\delta,-\beta^{\sigma}} x_{\delta-\beta^{\sigma}}+k_{\beta} c_{-\delta, \beta} x_{-\delta+\beta^{\sigma}} .
\end{aligned}
$$

Computing now the product $(9)\left[Q_{\gamma}, V_{\varphi}\right]$ but taking the same roots of the previous calculation $\gamma=\delta$ and $\varphi=\beta$, we have

(9) $\left[Q_{\delta}, V_{\beta}\right]$

$$
\begin{aligned}
= & (-1) c_{\delta, \beta} U_{(\delta+\beta)} \\
& +k_{\beta} c_{\delta, \beta^{\sigma}} x_{\delta+\beta^{\sigma}}+k_{\delta} c_{\delta^{\sigma}, \beta} x_{\delta^{\sigma}+\beta}-k_{\delta} c_{-\delta^{\sigma},-\beta} x_{-\delta^{\sigma}-\beta}-k_{\beta} c_{-\delta,-\beta^{\sigma}} x_{-\delta-\beta^{\sigma}} \\
& +(-1) c_{\delta,-\beta} U_{(\delta-\beta)} \\
& +k_{\delta} c_{\delta^{\sigma},-\beta} x_{\delta^{\sigma}-\beta}-k_{\delta} c_{-\alpha^{\sigma}, \beta} x_{-\alpha^{\sigma}+\beta}+k_{\beta} c_{\alpha,-\beta^{\sigma}} x_{\alpha-\beta^{\sigma}}-k_{\beta} c_{-\alpha, \beta^{\sigma}} x_{-\alpha+\beta^{\sigma}} .
\end{aligned}
$$

Then by computing the difference of the two obtained expressions we get:

$$
(5)-(9) \quad 2 c_{\delta, \beta} U_{(\delta+\beta)}+H_{1}=\left[P_{\delta}, U_{\beta}\right]-\left[Q_{\delta}, V_{\beta}\right] .
$$

In fact, the respective second lines in (5) and (9) are equal so they cancel, while the two last ones are opposite so they add to twice that line, which can be simplified to

$$
H_{1}=2\left(k_{\delta} c_{\delta^{\sigma},-\beta}\left(x_{-\delta^{\sigma}+\beta}-x_{\delta^{\sigma}-\beta}\right)-k_{\beta} c_{\delta,-\beta^{\sigma}}\left(x_{\delta-\beta^{\sigma}}-x_{-\delta+\beta^{\sigma}}\right)\right) .
$$

Proceeding similarly with the products (6) and (8) taking the same pair of roots in each of them, namely $\delta=\gamma$ and $\beta=\varphi$, one obtains

$$
\begin{gathered}
(6)+(8) \quad 2 c_{\gamma, \varphi} V_{(\gamma+\varphi)}+T_{2}=\left[P_{\gamma}, V_{\varphi}\right]+\left[Q_{\gamma}, U_{\varphi}\right] \\
T_{2}=2 i\left(k_{\gamma} c_{\gamma^{\sigma},-\varphi}\left(x_{\gamma^{\sigma}-\varphi}+x_{-\gamma^{\sigma}+\varphi}\right)-k_{\varphi} c_{\gamma,-\varphi^{\sigma}}\left(x_{\gamma-\varphi^{\sigma}}+x_{-\gamma+\varphi^{\sigma}}\right)\right) .
\end{gathered}
$$


8.3. Brackets of fields. Recalling 8.2 and the definitions 4.4 we take bases of $p_{0, \lambda}$ and $p_{0, \mu}$ respectively. That is,

$$
\begin{aligned}
& \Xi_{\mathfrak{p}}(\lambda)=\left\{W_{\eta}, U_{\delta}, V_{\gamma}: \eta \in \rho^{-1}(\lambda)_{\mathbb{R}}, \delta, \gamma \in \rho^{-1}(\lambda)_{\mathbb{C}}^{*}\right\}, \\
& \Xi_{\mathfrak{p}}(\mu)=\left\{W_{\alpha}, U_{\beta}, V_{\varphi}: \alpha \in \rho^{-1}(\mu)_{\mathbb{R}}, \beta, \varphi \in \rho^{-1}(\mu)_{\mathbb{C}}^{*}\right\} .
\end{aligned}
$$

With these two basis we may form the corresponding local fields and with them nine brackets. However, we shall need only those contained in the following table. Using (5.4) (for $\eta, \alpha$ real and $\gamma, \delta, \beta$ and $\varphi$ complex) they are:

(1) $\left[W_{\eta}^{F}, W_{\alpha}^{F}\right]=\left(\frac{-1}{\lambda(E)}\right) \operatorname{Ta}\left(\left[R_{\eta}, W_{\alpha}\right]\right)-\left(\frac{-1}{\mu(E)}\right) T a\left(\left[R_{\alpha}, W_{\eta}\right]\right)$,

(5) $\quad\left[U_{\delta}^{F}, U_{\beta}^{F}\right]=\left(\frac{-1}{\lambda(E)}\right) T a\left(\left[P_{\delta}, U_{\beta}\right]\right)-\left(\frac{-1}{\mu(E)}\right) T a\left(\left[P_{\beta}, U_{\delta}\right]\right)$,

(6) $\left[U_{\delta}^{F}, V_{\varphi}^{F}\right]=\left(\frac{-1}{\lambda(E)}\right) \operatorname{Ta}\left(\left[P_{\delta}, V_{\varphi}\right]\right)-\left(\frac{-1}{\mu(E)}\right) \operatorname{Ta}\left(\left[Q_{\varphi}, U_{\delta}\right]\right)$,

(8) $\left[V_{\gamma}^{F}, U_{\beta}^{F}\right]=\left(\frac{-1}{\lambda(E)}\right) \operatorname{Ta}\left(\left[Q_{\gamma}, U_{\beta}\right]\right)-\left(\frac{-1}{\mu(E)}\right) \operatorname{Ta}\left(\left[P_{\beta}, V_{\gamma}\right]\right)$,

$$
\left[V_{\gamma}^{F}, V_{\varphi}^{F}\right]=\left(\frac{-1}{\lambda(E)}\right) \operatorname{Ta}\left(\left[Q_{\gamma}, V_{\varphi}\right]\right)-\left(\frac{-1}{\mu(E)}\right) \operatorname{Ta}\left(\left[Q_{\varphi}, V_{\gamma}\right]\right) .
$$

Now we consider the following quantities for $\delta \in \rho^{-1}(\lambda)_{\mathbb{C}}^{*}$ and $\varphi \in \rho^{-1}(\mu)_{\mathbb{C}}^{*}$ :

$$
\begin{aligned}
D(\delta, \varphi) & =\left[U_{\delta}^{F}, U_{\varphi}^{F}\right](E)-\left[V_{\delta}^{F}, V_{\varphi}^{F}\right](E), \\
S(\delta, \varphi) & =\left[U_{\delta}^{F}, V_{\varphi}^{F}\right](E)+\left[V_{\delta}^{F}, U_{\varphi}^{F}\right](E) .
\end{aligned}
$$

We have

$$
\begin{aligned}
D(\delta, \varphi)= & \left(\frac{-1}{\lambda(E)}\right)\left\{\operatorname{Ta}\left[P_{\delta}, U_{\varphi}\right]-T a\left[Q_{\delta}, V_{\varphi}\right]\right\} \\
& -\left(\frac{-1}{\mu(E)}\right)\left\{\operatorname{Ta}\left[P_{\varphi}, U_{\delta}\right]-T a\left[Q_{\varphi}, V_{\delta}\right]\right\}, \\
S(\delta, \varphi)= & \left(\frac{-1}{\lambda(E)}\right)\left\{\operatorname{Ta}\left[P_{\delta}, V_{\varphi}\right]+T a\left[Q_{\delta}, U_{\varphi}\right]\right\} \\
& -\left(\frac{-1}{\mu(E)}\right)\left\{T a\left[Q_{\varphi}, U_{\delta}\right]+T a\left[P_{\varphi}, V_{\delta}\right]\right\} .
\end{aligned}
$$

8.4. Study of $D(\delta, \varphi)$. By 8.3 we have

$$
\begin{aligned}
& \left\{T a\left[P_{\delta}, U_{\varphi}\right]-T a\left[Q_{\delta}, V_{\varphi}\right]\right\}=2 c_{\delta, \varphi} U_{(\delta+\varphi)}+T a\left(H_{1}\right), \\
& \left\{T a\left[P_{\varphi}, U_{\delta}\right]-T a\left[Q_{\varphi}, V_{\delta}\right]\right\}=2 c_{\varphi, \delta} U_{(\delta+\varphi)}+T a\left(H_{1}^{*}\right),
\end{aligned}
$$

where $H_{1}^{*}$ is just $H_{1}$ in $(8.4$ but with $\delta$ and $\varphi$ interchanged. That is,

$$
H_{1}^{*}=2\left(k_{\varphi} c_{\varphi^{\sigma},-\delta}\left(x_{-\varphi^{\sigma}+\delta}-x_{\varphi^{\sigma}-\delta}\right)-k_{\delta} c_{\varphi,-\delta^{\sigma}}\left(x_{\varphi-\delta^{\sigma}}-x_{-\varphi+\delta^{\sigma}}\right)\right) .
$$

Now we observe that the equalities

$$
-c_{\alpha,-\varphi^{\sigma}}=c_{-\varphi^{\sigma}, \alpha}=c_{\varphi^{\sigma},-\alpha}, \quad-c_{\varphi,-\alpha^{\sigma}}=c_{-\alpha^{\sigma}, \varphi}=c_{\alpha^{\sigma},-\varphi}
$$


clearly yield $H_{1}=H_{1}^{*}$. Since $c_{\alpha, \varphi}=-c_{\varphi, \alpha}$, we have that $D(\delta, \varphi)$ is

$$
\begin{aligned}
D(\delta, \varphi)= & \left(\frac{-1}{\lambda(E)}\right)\left(2 c_{\delta, \varphi} U_{(\delta+\varphi)}+T a\left(H_{1}\right)\right) \\
& -\left(\frac{-1}{\mu(E)}\right)\left(2 c_{\varphi, \delta} U_{(\delta+\varphi)}+T a\left(H_{1}^{*}\right)\right) \\
= & 2 c_{\delta, \varphi} U_{(\delta+\varphi)}\left(\left(\frac{-1}{\lambda(E)}\right)+\left(\frac{-1}{\mu(E)}\right)\right) \\
& +\left(\left(\frac{-1}{\lambda(E)}\right)-\left(\frac{-1}{\mu(E)}\right)\right)\left(T a\left(H_{1}\right)\right),
\end{aligned}
$$

and setting now, for the involved functions of $(\lambda, \mu, \delta, \varphi)$ and $(\lambda, \mu)$, the notations

$$
\begin{aligned}
\Theta_{(\lambda, \mu, \delta, \varphi)} & =\left(2 c_{\delta, \varphi}\right)\left(\frac{\lambda(E)+\mu(E)}{\lambda(E) \mu(E)}\right) \neq 0, \\
\Lambda_{(\lambda, \mu)} & =\left(\frac{\lambda(E)-\mu(E)}{\lambda(E) \mu(E)}\right),
\end{aligned}
$$

we have for $D(\delta, \varphi)$ the expression

$$
D(\delta, \varphi)=\Theta_{(\lambda, \mu, \delta, \varphi)} U_{(\delta+\varphi)}+\Lambda_{(\lambda, \mu)}\left(T a\left(H_{1}\right)\right) .
$$

8.5. Study of $S(\delta, \varphi)$. Let us consider now $S(\delta, \varphi)$ in (8.7), for which we have to use (8.5) and (8.6) (with $\delta$ instead of $\gamma$ ).

$$
\begin{aligned}
& T a\left[P_{\delta}, V_{\varphi}\right]+T a\left[Q_{\delta}, U_{\varphi}\right]=2 c_{\delta, \varphi} V_{(\delta+\varphi)}+T a\left(T_{2}\right), \\
& T a\left[Q_{\varphi}, U_{\delta}\right]+T a\left[P_{\varphi}, V_{\delta}\right]=2 c_{\varphi, \delta}\left(V_{(\delta+\varphi)}\right)+T a\left(T_{2}^{*}\right) .
\end{aligned}
$$

As above, $T_{2}^{*}$ is just $T_{2}$ (8.6) (with $\delta$ and $\varphi$ interchanged). That is,

$$
T_{2}^{*}=2 i k_{\varphi} c_{\varphi^{\sigma},-\delta}\left(x_{\varphi^{\sigma}-\delta}+x_{\delta-\varphi^{\sigma}}\right)-2 i k_{\delta} c_{\varphi,-\delta^{\sigma}}\left(x_{\varphi-\delta^{\sigma}}+x_{\delta^{\sigma}-\varphi}\right) .
$$

Now we observe that $c_{\delta,-\varphi^{\sigma}}=-c_{-\varphi^{\sigma}, \delta}=-c_{\varphi^{\sigma},-\delta}$ and $c_{\delta^{\sigma},-\varphi}=-c_{-\varphi, \delta^{\sigma}}=$ $-c_{\varphi,-\delta^{\sigma}}$, which clearly yield $T_{2}=T_{2}^{*}$, and in turn $S(\delta, \varphi)$ becomes

$$
\begin{aligned}
S(\delta, \varphi)= & \left(\frac{-1}{\lambda(E)}\right)\left(2 c_{\delta, \varphi} V_{(\delta+\varphi)}+T a\left(T_{2}\right)\right) \\
& -\left(\frac{-1}{\mu(E)}\right)\left(2 c_{\varphi, \delta} V_{(\delta+\varphi)}+T a\left(T_{2}\right)\right) \\
= & 2 c_{\delta, \varphi} V_{(\delta+\varphi)}\left(\left(\frac{-1}{\lambda(E)}\right)+\left(\frac{-1}{\mu(E)}\right)\right) \\
& +\left(\left(\frac{-1}{\lambda(E)}\right)-\left(\frac{-1}{\mu(E)}\right)\right)\left(T a\left(T_{2}\right)\right),
\end{aligned}
$$

which, using again notation 8.8 , is

$$
S(\delta, \varphi)=\Theta_{(\lambda, \mu, \delta, \varphi)} V_{(\delta+\varphi)}+\Lambda_{(\lambda, \mu)} T a\left(T_{2}\right) .
$$


8.5.1. Resulting formulae. We write formulae 8.9 and 8.10 for the roots $\lambda, \mu \in$ $\Omega \subset \Phi^{+}\left(\mathfrak{g}_{0}, \mathfrak{a}_{0}\right), \delta \in \rho^{-1}(\lambda)_{\mathbb{C}}^{*}$, and $\varphi \in \rho^{-1}(\mu)_{\mathbb{C}}^{*}:$

$$
\begin{gathered}
\Theta_{(\lambda, \mu, \delta, \varphi)} U_{(\delta+\varphi)}+\Lambda_{(\lambda, \mu)}\left(T a\left(H_{1}\right)\right)=\left[U_{\delta}^{F}, U_{\varphi}^{F}\right](E)-\left[V_{\delta}^{F}, V_{\varphi}^{F}\right](E), \\
\Theta_{(\lambda, \mu, \delta, \varphi)} V_{(\delta+\varphi)}+\Lambda_{(\lambda, \mu)}\left(T a\left(T_{2}\right)\right)=\left[U_{\delta}^{F}, V_{\varphi}^{F}\right](E)+\left[V_{\delta}^{F}, U_{\varphi}^{F}\right](E) .
\end{gathered}
$$

We have to add also the case in which $(\delta+\varphi)$ is real and both $\delta$ and $\varphi$ complex. Again $\lambda, \mu \in \Omega \subset \Phi^{+}\left(\mathfrak{g}_{0}, \mathfrak{a}_{0}\right), \delta \in \rho^{-1}(\lambda)_{\mathbb{C}}^{*}$, and $\varphi \in \rho^{-1}(\mu)_{\mathbb{C}}^{*}$. In this case from 8.11 and having 4.5 in mind we have:

$$
\begin{aligned}
& k_{(\delta+\varphi)}=1, \\
& \Theta_{(\lambda, \mu, \delta, \varphi)} W_{(\delta+\varphi)}+\Lambda_{(\lambda, \mu)}\left(T a\left(H_{1}\right)\right)=\left[U_{\delta}^{F}, U_{\varphi}^{F}\right](E)-\left[V_{\delta}^{F}, V_{\varphi}^{F}\right](E), \\
& k_{(\delta+\varphi)}=(-1), \\
& \Theta_{(\lambda, \mu, \delta, \varphi)} W_{(\delta+\varphi)}+\Lambda_{(\lambda, \mu)}\left(T a\left(T_{2}\right)\right)=\left[U_{\delta}^{F}, V_{\varphi}^{F}\right](E)+\left[V_{\delta}^{F}, U_{\varphi}^{F}\right](E) .
\end{aligned}
$$

We need to consider also the case in which both $\delta$ and $\varphi$ are real. That is, $\lambda$, $\mu \in \Omega \subset \Phi^{+}\left(\mathfrak{g}_{0}, \mathfrak{a}_{0}\right), \delta \in \rho^{-1}(\lambda)_{\mathbb{R}}$, and $\varphi \in \rho^{-1}(\mu)_{\mathbb{R}}$. Furthermore, the first line in 8.1 in the present case clearly yields $k_{\delta} k_{\varphi}=k_{(\delta+\varphi)}$ and then formulae 8.12 become:

$$
\begin{aligned}
& k_{(\delta+\varphi)}=1, \quad k_{\delta}=k_{\varphi}=1, \\
& \Theta_{(\lambda, \mu, \delta, \varphi)} W_{(\delta+\varphi)}+\Lambda_{(\lambda, \mu)}\left(T a\left(H_{1}\right)\right)=\left[W_{\delta}^{F}, W_{\varphi}^{F}\right](E), \\
& k_{(\delta+\varphi)}=1, \quad k_{\delta}=k_{\varphi}=-1, \\
& \Theta_{(\lambda, \mu, \delta, \varphi)} W_{(\delta+\varphi)}+\Lambda_{(\lambda, \mu)}\left(T a\left(H_{1}\right)\right)=-\left[W_{\delta}^{F}, W_{\varphi}^{F}\right](E), \\
& k_{(\delta+\varphi)}=(-1), \quad k_{\delta}=1, \quad k_{\varphi}=-1, \\
& \Theta_{(\lambda, \mu, \delta, \varphi)} W_{(\delta+\varphi)}+\Lambda_{(\lambda, \mu)}\left(T a\left(T_{2}\right)\right)=\left[W_{\delta}^{F}, W_{\varphi}^{F}\right](E), \\
& k_{(\delta+\varphi)}=(-1), \quad k_{\delta}=-1, \quad k_{\varphi}=1, \\
& \Theta_{(\lambda, \mu, \delta, \varphi)} W_{(\delta+\varphi)}+\Lambda_{(\lambda, \mu)}\left(T a\left(T_{2}\right)\right)=\left[W_{\delta}^{F}, W_{\varphi}^{F}\right](E) .
\end{aligned}
$$

\section{REFERENCES}

[1] Berndt, J., Console, S., Olmos, C. Submanifolds and Holonomy. Second edition. CRC Press, Boca Raton, 2016. MR 3468790

[2] Freudenthal, H., de Vries, H. Linear Lie Groups. Academic Press, New York and London, 1969. MR 0260926.

[3] Heintze, E., Olmos, C., Thorbergsson, G. Submanifolds with constant principal curvatures and normal holonomy groups. Internat. J. Math. 2 (1991), no. 2, 167-175. MR 1094702

[4] Helgason S. Differential Geometry, Lie Groups and Symmetric Spaces. Academic Press, New York and London, 1978. MR 0514561.

[5] Kammeyer, H. An explicit rational structure for real semisimple Lie algebras. J. Lie Theory 24 (2014), no. 2, 307-319. MR 3235892

[6] Knapp, A. Lie Groups Beyond an Introduction. Second edition. Birkhäuser, Boston, 2002. MR 1920389. 
[7] Thorbergsson, G. Isoparametric foliations and their buildings. Ann. Math. (2) 133 (1991), no. 2, 429-446. MR 1097244

[8] Warner, G. Foundation of Differentiable Manifolds and Lie Groups. Scott, Foresman and Co. Glenview, Ill.-London, 1971. MR 0295244.

Cristián U. Sánchez

Fa.M.A.F. Universidad Nacional de Córdoba and C.I.E.M. CONICET, Medina Allende s/n, Ciudad Universitaria, 5000 Córdoba, Argentina

csanchez@famaf .unc.edu.ar

Received: May 31, 2018

Accepted: April 11, 2019 